\title{
Shigella: A Highly Virulent and Elusive Pathogen
}

\author{
Mussaret Bano Zaidi • Teresa Estrada-García
}

Published online: 25 March 2014

(C) Springer International Publishing AG 2014

\begin{abstract}
Despite a significant decrease in Shigella-related mortality, shigellosis continues to carry a significant burden of disease worldwide, particularly in Asia and Africa. Shigella is a highly virulent pathogen comprised of four major species with numerous subtypes. Shigella dysenteriae and Shigella flexneri infections are predominant in resource-limited settings. Clinical presentations range from mild watery diarrhea to severe dysentery with systemic complications such as electrolyte imbalance, seizures and hemolytic uremic syndrome. S. dysenteriae subtype 1, the producer of Shiga toxin, causes the most severe illness and highest mortality. Susceptible strains of Shigella may be effectively treated with inexpensive oral antibiotics such as ampicillin or trimethoprimsulfamethoxazole. Unfortunately, multidrug-resistant strains have emerged that have rendered most antibiotics, including fluoroquinolones and extended-spectrum cephalosporins, ineffective. Management and prevention of shigellosis represents a major public health challenge. The development of an effective vaccine is urgently needed to decrease its global impact.
\end{abstract}

Keywords Shigella $\cdot$ Morbidity $\cdot$ Mortality $\cdot$ Antimicrobial resistance $\cdot$ Epidemiology $\cdot$ Socioeconomic conditions · Virulence factors $\cdot$ Vaccine $\cdot$ Developing countries . Industrialized countries · Type III secretion system - Serotype

M. B. Zaidi $(\bowtie)$

Microbiology Research Laboratory, Hospital General O'Horan, Av. Itzaes x Jacinto Canek, 97000 Mérida, Mexico

e-mail: mbzaidi@prodigy.net.mx

M. B. Zaidi

Infectious Diseases Research Unit, Hospital Regional de Alta Especialidad de la Península de Yucatán, Calle 7 No. 433 x 20 y 22, Fracc. Altabrisa, 97130 Merida, Mexico

T. Estrada-García

Department of Molecular Biomedicine, CINVESTAV-IPN, Av. Instituto Politecnico Nacional 2508, San Pedro Zacatenco, 07360 Mexico City, Mexico

e-mail: testrada@cinvestav.mx switching · Immune evasion · Fluoroquinolones · Extended-spectrum cephalosporins $\cdot$ Ipa $\cdot$ Tropical medicine $\cdot$ Bacterial

\section{Introduction}

While child mortality has markedly decreased in recent decades, diarrheal disease remains the second leading cause of mortality among children under five years of age [1]. Shigella, a highly virulent pathogen that causes bacterial dysentery, is one of the leading causes of diarrheal disease and contributes significantly to the burden worldwide. Despite numerous efforts directed at its prevention and control, that goal remains elusive. This paper will discuss important features of the epidemiology, pathogenesis, and clinical presentation of shigellosis and highlight recent trends and developments relevant to its prevention and management.

\section{Shigella- Related Mortality, but not Morbidity, has Decreased Substantially Over the Last 50 Years}

Shigellosis has undergone significant changes over recent decades. In 1999, Kotloff et al. [2] estimated that 165 million cases of shigellosis and 1.1 million shigellosis-related deaths, most of which occurred in Asia, occurred annually between 1966 and 1997. However, a recent review for the 1990 to 2009 time period [3] found that while the total number of shigellosis episodes in Asia had negligibly decreased, the total number of shigellosis-related deaths substantially decreased to approximately 14,000 per year. The authors proposed that the decrease in Shigella-related deaths could be a consequence of the widespread implementation of non-specific interventions such as measles vaccination, vitamin A supplementation, and improved nutrition. These results concur with a comprehensive study from China [4] that found a 3-fold decrease in morbidity and a 10-fold to 31-fold decrease in mortality from bacillary dysentery in China from 1991 to 2000. Another 
factor that may have contributed to a worldwide reduction in Shigella-related deaths could be the still-unexplained decrease in Shigella dysenteriae type 1 (SD1) over the last decades. This serotype, a cause of large-scale dysentery outbreaks and high case fatality rates, was widespread in Latin America, Asia, and Africa two to four decades ago [5-8]. Currently, SD1 infections are primarily reported on the Indian subcontinent, although outbreaks and sporadic infections are also reported in Africa [9-12].

Despite the substantial decrease in mortality, the global burden of shigellosis remains considerable. In a large prospective case-control study in Africa and Asia [13], Shigella was isolated from $17 \%, 66 \%$ and $78 \%$, respectively, of infants, toddlers, and children with moderate-to-severe dysentery, and was among the top four pathogens associated with moderate-to-severe diarrhea at all study sites. A large multicenter study in six Asian countries [14] found an overall incidence of 2.1 episodes/1000 residents/year for all ages. Incidence rates were highest in children less than five years of age (13.2 episodes/1000/y) followed by those 70 years and over ( 2 episodes/1000/y). The incidence of shigellosis was highest in children from Bangladesh (48.2/ $1000 / y)$ and lowest in children from Thailand (4.0/1000/y). Shigellosis is prevalent in Latin American countries as well. Kosek [15] reported an $8.3 \%$ isolation rate in children with diarrhea from the Peruvian Amazon, while our group isolated Shigella from $4.5 \%$ of infants less than three years of age from a rural community in Yucatan, Mexico [16]. A higher prevalence of Shigella-associated diarrhea has been reported in hospital settings. Two hospital-based studies in Brazil isolated Shigella in $10 \%$ of diarrheal stools from children $[17,18]$. During the last three years we have witnessed an upsurge of severe shigellosis at our hospital center in Mexico where it is the cause of acute diarrheal episodes in $11 \%$ of children [19•].

\section{Shigella is a Highly Virulent Organism}

Shigella is a gram-negative intracellular bacterial pathogen that initiates infection by invading cells and causing intense inflammation in the colonic and rectal epithelium. A low infective dose on the order of 10 to 100 organisms is sufficient to produce disease. It is typically transmitted by contaminated food and water or by direct contact with an infected person. Shigella species possess a large virulence plasmid that carries the genes necessary for cell invasion, including those for a type III secretion system (TTSS) used to gain entry into epithelial cells [20, 21]. The ipa-ipg operon encodes for important effector/translocator proteins (IpaA through IpaD) that are injected into the host cell by the TTSS. Once Shigella enters the epithelial cell, it escapes from the phagocyte vacuole and actively proliferates within the cytosol of infected cells (Fig. 1) [22, 23]. Recently, Paciello et al. [24•] showed that Shigella modifies its membrane lipopolysaccharide (LPS) composition in order to evade pathogen recognition and eradication processes.

In addition to these virulence factors, Shigella also may produce one or more of several toxins. Two enterotoxins that cause fluid secretion in animal models [25] have been identified: Shigella enterotoxin 1 (ShET-1) and Shigella enterotoxin 2 (ShET-2). ShET1, predominantly found in S. flexneri, is encoded by the chromosomal gene set. It is a classical AB toxin comprised of several B subunits that bind to specific molecules on the target cell and a single A subunit that carries out the toxic enzymatic reaction within the cell. ShET-2 is encoded by a plasmid-borne gene sen and may be expressed by all Shigella species [26, 27]. Aside from an enterotoxic activity similar to that of ShET-1, ShET-2 is also believed to induce inflammation of epithelial cells via IL-8 secretion [28].

Shiga toxin 1(Stx 1), the most widely known Shigella toxin, is produced by SD1. Responsible for the most severe manifestations of shigellosis, Stx1 has an AB5 subunit structure. The five B subunits bind Shiga toxin to the glycolipid $\mathrm{Gb}_{3}$ receptor present on target cells such as intestinal villi, glomerular endothelial cells, mesangial cells, podocytes, and renal tubular cells [29]. The A subunit is a cytotoxic protein that acts on the 28S rRNA component of eukaryotic ribosomes, leading to protein synthesis inhibition and destruction of endothelial cells [30]. It is also believed to increase the expression of chemokines and cytokines, which in turn leads to chemoattraction and activation of neutrophils, and ultimately, to the binding of inflammatory cells to the endothelium [31].

\section{Shigella Species Shift with Changing Socioeconomic Conditions}

Shigella is comprised of four major species: S. dysenteriae, Shigella flexneri, Shigella boydii, and Shigella sonnei. With the exception of $S$. sonnei, each species is further subdivided into serotypes. S. dysenteriae has 15 ; $S$. flexneri has six, and $S$. boydii has 18 serotypes [32] that are based on the structure of exposed terminal $\mathrm{O}$ polysaccharides that form part of their outer membrane LPS [33]. Several investigators have noted an association between Shigella species and socioeconomic conditions [34•, 35]. S. dysenteriae and S. flexneri, associated with poverty and poor hygiene, are the predominant species in resource-limited settings, while $S$. sonnei is more common in affluent regions [35-37]. Presently, SD1 is endemic and epidemic on the Indian subcontinent [9, 10, 38], while $S$. flexneri is predominant throughout Southeast Asia [14, 34•, 39, 40], Latin America [15, 17, 19•], and Africa [12, 41]. A shift in the dominant infecting species from $S$. flexneri to $S$. sonnei has been documented in countries that have undergone recent improvements in economic status such as China, Vietnam, and Thailand [14, 34•, 40]. The persistence 


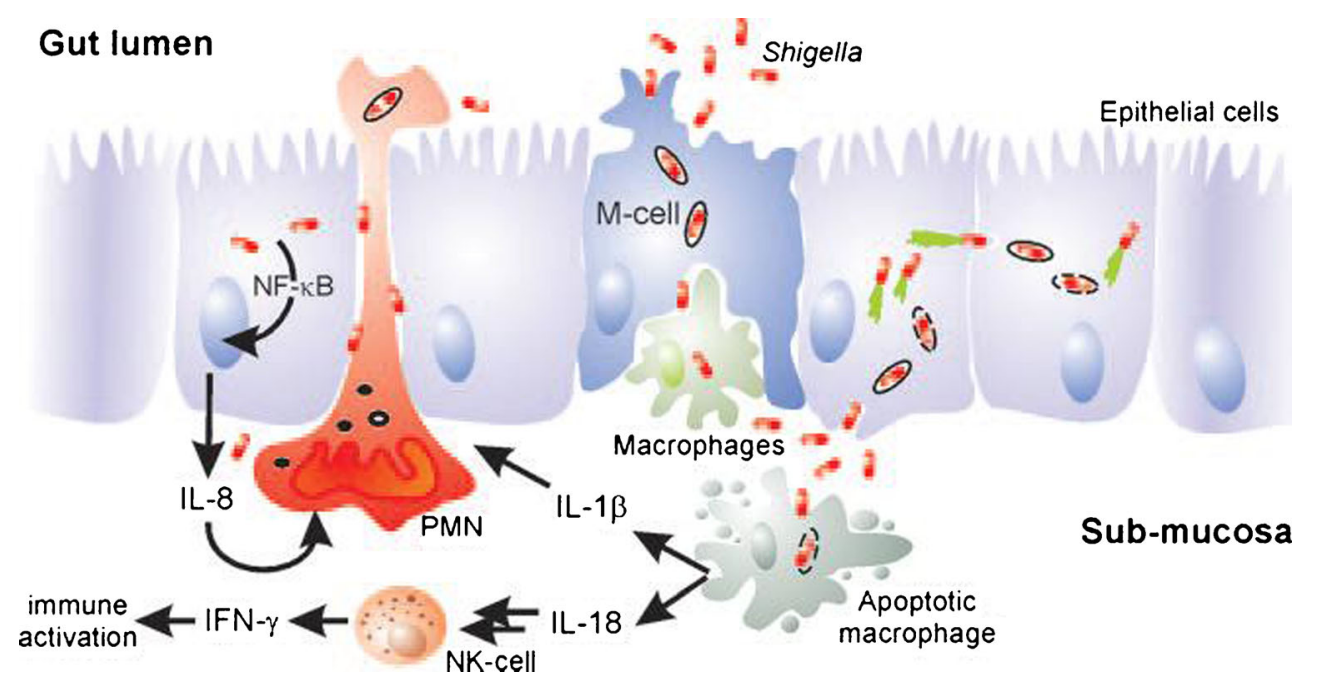

Fig. 1 In the colonic mucosa, Shigella is transcytosed across M cells into the underlying gut-associated lymphoid tissues. They later enter macrophages and induce apoptosis, leading to release of the bacteria on the basal side of the epithelium. Upon receipt of a secretion signal, Shigella invades epithelial cells from the basolateral side and spreads to adjacent cells. Proinflammatory signaling by host cells further activates the

of $S$. flexneri in Latin America likely reflects the prevailing poverty in the region. In industrialized countries, Shigella infections have frequently been found in day care centers or linked to consumption of imported food [42-44].

\section{Serotype Switching has Important Implications for Vaccine Development}

During natural infection, Shigella elicits an antibodymediated, serotype-specific immune response. Serotype switching through $\mathrm{O}$-antigenic variation is a common strategy used by the organism to evade host immunity [45]. The replacement of commonly circulating serotypes by newly emerging ones in response to selective pressure from population immunity has been well documented in China where this phenomenon decreased the effectiveness of a $S$. flexneri $2 \mathrm{a}-$ based vaccine $[34 \cdot, 46]$.

There is consensus within the scientific community that a global Shigella vaccine must protect against SD1, S. sonnei, and all $S$. flexneri serotypes [47]. Various candidate vaccines have been developed and tested in clinical trials including killed bacteria, live attenuated strains, and polysaccharide conjugates and LPS-protein mixtures. Due to poor immunogenicity, limited scope, or strong adverse reactions, however, none of these has been entirely satisfactory for large-scale use [47-49]. More recently, IpaB and IpaD, two type-III secretion proteins that are highly immunogenic and conserved across Shigella species, have been tested for their use in crossprotective vaccines $[50,51]$. Intranasal immunization with a subcellular vaccine prepared from pathogenic $S$. flexneri immune response, which initially exacerbates infection and tissue destruction. Ultimately, the infection is resolved when PMN phagocytose and kill the invading Shigella. (Reproduced with permission from Schroeder GN, Hilbi H. Molecular Pathogenesis of Shigella spp.: Controlling Host Cell Signaling, Invasion, and Death by Type III Secretion Clin. Microbiol. Rev. 2008; 21: 134-156)

composed of LPS, IpaB, IpaC and IpaD was well tolerated and induced strong gastrointestinal mucosal $\operatorname{IgA}$ responses in $58 \%$ and plasma IgA responses in $50 \%$ of volunteers [50]. Despite these encouraging results, improvement will be necessary to achieve higher conversion rates and demonstrate protective efficacy.

\section{The Clinical Presentation of Shigella Infections is Very Variable and Depends on the Infecting Species}

Shigellosis is typically an invasive infection of the human colon and rectum, causing severe inflammation and tissue necrosis. Clinical presentations, nonetheless, are highly variable. Some patients have a mild, watery diarrhea with no fever, while others have severe dysentery, high fever, and systemic complications [14, 19•, 40, 52•]. Shigella infections may also lead to persistent diarrhea in young children [14]. Although all four Shigella species may cause both intestinal and extra-intestinal disease, SD1 is associated with the greatest severity. In Bangladesh [52•], children infected with SD1 more frequently had grossly bloody stools, abdominal tenderness, leukemoid reaction, and rectal prolapse when compared to children infected by $S$. dysenteriae serotypes 210 or other Shigella species. Seizures have also been reported worldwide [14, 19•, 40, 52•, 53] and are significantly associated with death in impoverished regions [52•]. The rate of seizures is highly variable, especially by region. Extremely high rates have been reported in Thailand $(27.5 \%)$ [53]. In Bangladesh [52•], seizures have been documented in $8 \%$ of children with $S$. dysenteriae infections (all serotypes) and $5 \%$ 
of $S$. flexneri infections, but in none of the infections caused by $S$. sonnei and S. boydii. In Mexico [19•], $11 \%$ of our hospitalized children with Shigella gastroenteritis presents with seizures, almost invariably caused by $S$. flexneri. Although most seizures occur with $S$. dysenteriae and $S$. flexneri infections, seizures and severe disease manifestations have also been reported for $S$. sonnei [40, 54•]. In a 14year study conducted at two large hospitals in Vietnam, Vinh et al. [40] found that $7.3 \%$ of children infected with $S$. flexneri developed seizures compared to $14.3 \%$ of those infected with S. sonnei.

Hemolytic uremic syndrome (HUS), comprised of the triad of hemolytic anemia, thrombocytopenia, and renal insufficiency, occurs in about 8-13\% of dysenteric patients infected with SD1, with a case fatality rate of $36 \%$. It may occur, albeit rarely, with $S$. flexneri infections $[55,56]$. SD1 HUS typically develops one week or more after the onset of diarrhea. In contrast to $E$. coli O157:H7, it occurs after a course of bloody diarrhea with fever, almost exclusively in young children. Primarily due to the action of Shiga toxin on microvascular endothelial cells, most damage occurs in the kidneys and CNS [57]. End-stage renal disease and hypertension have also been reported, although less often than with STEC-related HUS [58]. Rare, but severe, complications include encephalopathy, hemiplegia, cardiomyopathy, and disseminated intravascular coagulation [58].

It should be noted that most published studies have been conducted in hospital settings, which leads to a selection bias towards greater severity. For example, $36 \%$ of children hospitalized for Shigella gastroenteritis at our center had a fever greater than $38.5^{\circ} \mathrm{C}$, compared to only $7 \%$ of young infants in a rural community in Yucatan, Mexico [16, 19•]. Dehydration and seizures were present, respectively, in $28 \%$ and $11 \%$ of the hospitalized children, but in none of the children from the community setting. Notably, the presence of grossly bloody stools was similar $(59 \%$ in hospitalized children compared to $47 \%$ in children from the community). In highly endemic settings where the population is repeatedly exposed to Shigella and other enteropathogens, protective immunity is acquired in infancy and tends to wane in old age. Susceptibility in elderly people, however, does not return to that of immunologically naïve infants [16].

\section{Increasing Multidrug Resistant Shigella is a Serious Public Health Threat}

Shigella can cause severe illness and persistent diarrhea; young infants, the elderly, and immunosuppressed individuals are at particular risk and require prompt antimicrobial treatment. Shigella dysentery may be effectively treated with inexpensive oral antibiotics such as ampicillin or trimethoprimsulfamethoxazole when strains are susceptible. Unfortunately, our capacity to effectively treat severe infections is being undermined by increasing resistance to antimicrobial compounds worldwide. At present, countries with the greatest Shigella burden also face greater challenges of multidrug resistance. A recent review [59॰] of antimicrobial resistance trends worldwide showed that quinolone resistance in the Asia-Africa region increased at a distressingly rapid rate over the last decade, and that the proportion of resistant strains is more than ten times the rate in Europe and America. Worldwide, strains are becoming resistant not only to first-line oral antibiotics such as ampicillin, trimethoprim-sulfamethoxazole, and tetracycline, but to broad-spectrum antibiotics as well $[14,40,60]$. Outbreaks of multidrug-resistant, fluoroquinolone-resistant SD1 strains have occurred in India and Bangladesh over the last decade [9, 10, 38], and fluoroquinolone-resistant $S$. flexneri has been reported from China [46, 59॰]. Ceftriaxone-resistant S. flexneri and S. sonnei have been reported from south Asia $[40,46,59 \cdot, 61]$ and the Middle East [62, 63]. In Latin America, most strains remain fully susceptible to fluoroquinolones and third-generation cephalosporins $[15,17,18,19 \bullet]$.

Ciprofloxacin, pivmecillinam, and ceftriaxone are currently recommended by the World Health Organization for the treatment of multiresistant Shigella strains [32]. Azithromycin is another therapeutic option, although its use has been associated with rapid development of resistance. At our hospital center in Mexico we have used 3-to-5-day regimens of azithromycin in children of all ages with bloody diarrhea; notable clinical improvement has been observed within 24 hours for most patients, even for those strains with MICs in the intermediate range. We strongly recommend that susceptibility to azithromycin be tested by agar or broth dilution, as we have observed discordant results using disk diffusion (authors' unpublished results). For children unable to tolerate oral dosing or those in hypovolemic shock, we have successfully used intravenous or intramuscular ceftriaxone. Oral extendedspectrum cephalosporins are less effective for the treatment of shigellosis; a high rate of clinical failure has been observed in both adults [64] and children [65] treated with cefixime.

SD1 infections merit special mention due to the association of Shigella-associated HUS and previous antimicrobial treatment. In both SD1 and Shiga toxin-producing Escherichia coli (STEC), the genes encoding Shiga toxin are chromosomally encoded and linked with lambdoid bacteriophage sequences. In contrast to STEC infections, in which the use of antibiotics induces lysogenesis and increased Shiga toxin expression leads to increased mortality, the prophage in SD1 strains is defective and unable to induce lysogenesis [66]. Although exposure of SD1 to antibiotics does not increase Shiga toxin expression via bacteriophage lysogenesis, extracellular concentrations may increase after antibiotic-mediated killing and bacterial lysis [67].

Previous studies reported an increased association between use of trimethoprim-sulfamethoxazole and ampicillin and subsequent development of HUS [55]. Lower rates of HUS were observed in patients who received ciprofloxacin, nalidixic 
acid, and ceftriaxone, although the poorer outcomes are believed to be related to clinical failures due to antibiotic resistance $[55,67]$. Data from clinical trials has shown that if antimicrobial therapy is administered within the first three days after onset of dysentery, the incidence of HUS is very low. Bennish et al. [67] analyzed the data for 378 individuals infected with SD1 who received antimicrobial treatment before 96 hours after the onset of symptoms, of which $93 \%$ received an antimicrobial agent to which the organism was susceptible. The risk of developing HUS was 0.0026 for all participants and 0.004 for children.

\section{Conclusions}

Despite numerous efforts at prevention and control, shigellosis continues to be an important cause of acute diarrhea and dysentery worldwide. Factors that contribute to the persistence of shigellosis worldwide are poor socioeconomic conditions, serotype switching, and increasing antimicrobial resistance. Major challenges for the future are the development of an effective vaccine that can protect against strains that produce clinically important disease and the prevention and containment of multidrug-resistant Shigella strains. The Ipa effector proteins are promising candidates for a subunit-based vaccine. Greater efforts are required to further contain the widespread resistance to fluoroquinolones and extended-spectrum cephalosporins.

Acknowledgments This work was supported in part by the National Council for Science and Technology, Mexico (CONACyT), Grant SALUD-2004-CO1-159 (MBZ), NIH grant U01A/0082110, and FD-U0011934, as well as funding from the Fundacion Mexicana para la Salud, Capitulo Peninsular.

\section{Compliance with Ethics Guidelines}

Conflict of Interest Mussaret Bano Zaidi and Teresa Estrada-García declare that they have no conflict of interest.

Human and Animal Rights and Informed Consent This article does not contain any studies with human or animal subjects performed by any of the authors.

\section{References}

Papers of particular interest, published recently, have been highlighted as:

- Of importance

1. Black RE, Cousens S, Johnson HL, Lawn JE, Rudan I, Bassan DG, et al. Global, regional, and national causes of child mortality in 2008: a systematic analysis. Lancet. 2008;375:1969-87.
2. Kotloff KL, Winickoff JP, Ivanoff B, Clemens JD, Swerdlow DL, Sansonetti PJ, et al. Global burden of Shigella infections: implications for vaccine development and implementation of control strategies. Bull World Health Organ. 1999;77:651-66.

3. Bardhan P, Faruque ASG, Naheed A, Sack D. Decrease in shigellosis-related deaths without Shigella spp.-specific interventions, Asia. Emerg Infect Dis. 2010;16:1718-23.

4. Wang X, Tao F, Xiao D, Lee H, Deen J, Gong J, et al. Trend and disease burden of bacillary dysentery in China (1991-2000). Bull World Health Organ. 2006;84:561-8.

5. Mata L, Gangarosa E, Caceres A, Perera D, Mejicanos M. Epidemic Shiga bacillus dysentery in Central America. I. Etiologic investigations in Guatemala, 1969. J Infect Dis. 1970;122:170-80.

6. Taylor DN, Bodhidatta L, Brown JE, Echeverria P, Kunanusont C, Naigowit P, et al. Introduction and spread of multi-resistant Shigella dysenteriae I in Thailand. Am J Trop Med Hyg. 1989;40(1):77-85.

7. Ries AA, Wells DG, Olivola D, Ntakibirora M, Nyandwi S, Ntibakivayo M, et al. Epidemic Shigella dysenteriae type 1 in Burundi: panresistance and implications for prevention. J Infect Dis. 1994;169:1035-41.

8. Ebright JR et al. Epidemic Shiga bacillus dysentery in Central Africa. Am J Trop Med Hyg. 1984;33:1192-7.

9. Dutta S, Dutta D, Dutta P, Matsushita S, Bhattacharya SK, Yoshida S. Shigella dysenteriae Serotype 1 Kolkata, India. Emerg Infect Dis. 2003;9:1471-4.

10. Bhattacharya SK, Sarkar K, Nair GB, Faruque ASG, Sack DA. Multidrug-resistant Shigella dysenteriae type 1 in south Asia. Lancet Infect Dis. 2003;3:755.

11. Bercion R, Demartin M, Recio C, Massamba PM, Frank T, Escriba JM, et al. Molecular epidemiology of multidrugresistant Shigella dysenteriae type 1 causing dysentery outbreaks in Central African Republic, 2003-2004. Trans R Soc Trop Med Hyg. 2006;100:1151-8.

12. Naik DG. Prevalence and antimicrobial susceptibility patterns of Shigella species in Asmara, Eritrea, northeast Africa. J Microbiol Immunol Infect. 2006;39:392-5.

13. Kotloff KL, Blackwelder WC, Nasrin D, Farag TH, Panchalingam $\mathrm{S}, \mathrm{Wu} \mathrm{Y}$, et al. Burden and aetiology of diarrhoeal disease in infants and young children in developing countries (the Global Enteric Multicenter Study, GEMS): a prospective, case-control study. Lancet. 2013;382:209-22.

14. Von Seidlein L, Kim DR, Ali M, Lee H, Wang XY, Thiem VD, et al. A multicentre study of Shigella diarrhea in six Asian countries: disease burden, clinical manifestations, and microbiology. PLoS Med. 2006;3:1556-69.

15. Kosek M, Peñatoro-Yori P, Pan WK, Paredes-Olortegui M, Gilman RH, Perez J, et al. Pediatrics. 2008;122:e541-9.

16. Zaidi MB, Campos FD, Estrada-Garcia T, Gutierrez F, Leon M, Chim R, et al. Burden and transmission of zoonotic foodborne disease in a rural community in Mexico. Clin Infect Dis. 2012;55: $51-60$.

17. Nunes MR, Magalhaes PP, Penna FJ, Nunes JMM, Mendes EN. Diarrhea associated with Shigella in children and susceptibility to antimicrobials. J Pediatr (Rio J). 2012;88:125-8.

18. Bernardes Sousa MA, Nogueira Mendes E, Birchal Collares G, Peret-Filho LA, Penna FJ, Prazeres MP. Shigella in Brazilian children with acute diarrhea: prevalence, antimicrobial resistance and virulence genes. Mem Inst Oswaldo Cruz. 2013;108:30-5.

19. Zaidi MB, Estrada MT, Campos FD, Chim R, Arjona F, Leon M, et al. Incidence, clinical presentation, and antimicrobial resistance trends in Salmonella and Shigella infections from children in Yucatan, Mexico. Front Microbiol. 2013;4:288. Epidemiological data from the last decade show an upsurge in severe Shigella infections in southeast Mexico. 
20. Menard R, Sansonetti P, Parsot C. The secretion of the Shigella flexneri Ipa invasion is activated by epithelial cells and controlled by IpaB and IpaD. EMBO J. 1994;13:5293-302.

21. Cossart P, Sansonetti PJ. Bacterial invasion: the paradigms of enteroinvasive pathogens. Science. 2004;304:242-8.

22. Clerc P, Ryter A, Mounier J, Sansonetti PJ. Plasmid-mediated intracellular multiplication of Shigella flexneri. Ann Inst Pasteur Microbiol. 1986;137A(3):315-20.

23. Sansonetti PJ, Ryter A, Clerc P, Maurelli AT, Mounier J. Multiplication of Shigella flexneri within HeLa cells: lysis of the phagocytic vacuole and plasmid-mediated contact hemolysis. Infect Immun. 1986;51:461-9.

24. Paciello I, Silipo A, Lembo-Fazio L, Curcuru L, Zumsteg A, Noel G, et al. Intracellular Shigella remodels its LPS to dampen the innate immune recognition and evade inflammasome activation. Proc Natl Acad Sci USA. 2013;110(51):20843. An interesting study that illustrates how Shigella changes its lipopolysaccharide to evade the host immune response.

25. Fasano A, Noriega FR, Liao FM, Wang W, Levine MM. Effect of Shigella enterotoxin 1 (ShET1) on rabbit intestine in vitro and in vivo. Gut. 1997;40:505-11.

26. Vargas M, Gascon J, de Anta MT J, Vila J. Prevalence of Shigella enterotoxins 1 and 2 from Shigella strains isolated from patients with traveler's diarrhea. J Clin Microbiol. 1999;37: 3608-11.

27. Niyogi SK, Vargas M, Vila J. Prevalence of the sat, set and sen genes among diverse serotypes of Shigella flexneri strains isolated from patients with acute diarrhoea. Clin Microbiol Infect. 2004 Jun;10(6):574-6.

28. Farfan MJ, Toro CS, Barry EM, Nataro JP. Shigella enterotoxin-2 is a type III effector that participates in Shigella-induced interleukin 8 secretion by epithelial cells. FEMS Immunol Med Microbiol. 2011;61:332-9.

29. Warnier M, Romer W, Geelen J, Lesieur J, Amessou M, van den Heuvel L, et al. Trafficking of Shiga toxin/Shiga-like toxin-1 in human glomerular microvascular endothelial cells and human mesangial cells. Kidney Int. 2006;70:2085-91.

30. O'Brien AD, Tesh VL, Donohue-Rolfe A, Jackson MP, Olsnes S, Sandvig K, et al. Shiga toxin: biochemistry, genetics, mode of action, and role in pathogenesis. Curr Top Microbiol Immunol. 1992;180:65-94.

31. Harrison LM, van den Hoogen C, van Haaften WC, Tesh VL. Chemokine expression in the monocytic cell line THP-1 in response to purified Shiga toxin 1 and/or lipopolysaccharides. Infect Immun. 2005;73:403-12.

32. World Health Organization. Guidelines for the control of shigellosis, including epidemics due to Shigella dysenteriae 1. Geneva: WHO; 2005.

33. Lindberg AA, Karnell A, Weintraub A. The lipopolysaccharide of Shigella bacteria as a virulence factor. Rev Infect Dis. 1991;13 Suppl 4:S279-84.

34. Chang Z, Lu S, Chen L, Jin Q, Yang J. Causative species and serotypes of shigellosis in mainland China: systematic review and meta-analysis. PLoS One. 2012;7:e52515. A comprehensive review and update of the causative agents of shigellosis in China from 2001 to 2010 that strongly suggest there is an association between prevalent species of Shigella and regional economic status.

35. Admoni O, Yaqupsky P, Golan A, Kenes Y, Schifroni G, Horowitz I. Epidemiological, clinical and microbiological features of shigellosis among hospitalized children in northern Israel. Scand J Infect Dis. 1995;27:139-44.

36. Sivapalasingam S, Nelson JM, Joyce K, Hoekstra M, Angulo FJ, Mintz ED. High prevalence of antimicrobial resistance among Shigella isolates in the United States tested by the National Antimicrobial Resistance Monitoring System from 1999 to 2002. Antimicrob Agents Chemother. 2006;50:49-54.
37. Vrints M, Mairiaux E, van Meervenne E, Collard JM, Bertrand S. Surveillance of antibiotic susceptibility patterns among Shigella sonnei strains isolated in Belgium during the 18-year period 1990 to 2007. J Clin Microbiol. 2009;47:1379-85.

38. Pazhani GP, Sarkar B, Ramamurthy T, Bhattacharya SK, Takeda Y, Niyogi SK. Clonal multidrug-resistant Shigella dysenteriae Type 1 strains associated with epidemic and sporadic dysenteries in Eastern India. Antimicrob Agents Chemother. 2004;48:681-4.

39. Chompook P, Samosornsuk S, von Seidlein L, Jitsanguansuk S, Sirima N, Sudjai S, et al. Estimating the burden of shigellosis in Thailand: 36-month population-based surveillance study. Bull World Health Organ. 2005;83:739-46.

40. Vinh H, Nhu NT, Nga TV, Duy PT, Campbell JI, Hoang NV, et al. A changing picture of shigellosis in southern Vietnam: shifting species dominance, antimicrobial susceptibility and clinical presentation. BMC Infect Dis. 2009;9:204.

41. Hiranrattana A, Mekmullica J, Chatsuwan T, Pancharoen C, Thisyakorn U. Childhood shigellosis at King Chulalongkorn Memorial Hospital, Bangkok, Thailand: a 5-year review (19962000). Southeast Asian J Trop Med Public Health. 2005;36:683-5.

42. Goldberg EM, Balamuth F, Desrochers CR, Mittal MK. Seizure and altered mental status in a 12-year-old child with Shigella sonnei gastroenteritis. Pediatr Emerg Care. 2011;27:135-7.

43. Tiruneh M. Serodiversity and antimicrobial resistance pattern of Shigella isolates at Gondar University Teaching Hospital, Northwest Ethiopia. Jpn J Infect Dis. 2009;62:93-7.

44. Lewis HC, Ethelberg S, Olsen KE, Nielsen EM, Lisby M, Madsen $\mathrm{SB}$, et al. Outbreaks of Shigella sonnei infections in Denmark and Australia linked to consumption of imported raw baby corn. Epidemiol Infect. 2009;137:326-34.

45. Kosak GK, MacDonald D, Landry L, Farber JM. Foodborne outbreaks in Canada linked to produce: 2001 through 2009. J Food Prot. 2013;76:173-83.

46. Mohle-Boetani JC et al. Communitywide shigellosis: control of an outbreak and risk factors in child day-care centers. Am J Public Health. 1995;85:812-6.

47. West NP, Sansonetti P, Mounier J, Exley RM, Parsot C, Guadagnini $\mathrm{S}$, et al. Optimization of virulence functions through glucosylation of Shigella LPS. Science. 2005;307:1313-7.

48. Ye C, Lan R, Xia S, Zhang J, Sun Q, Zhang S, et al. Emergence of a new multidrug-resistant serotype $\mathrm{X}$ variant in an epidemic clone of Shigella flexneri. J Clin Microbiol. 2010;48:419-26.

49. Levine MM, Kotloff K, Barry EM, Pasetti MF, Sztein MB. Clinical trials of Shigella vaccines: two steps forward and one step back on a long, hard road. Nat Rev Microbiol. 2007;5:540-53.

50. McKenzie R et al. Safety and immunogenicity of an oral, inactivated, whole-cell vaccine for Shigella sonnei: preclinical studies and a Phase I trial. Vaccine. 2006;24:3735-45.

51. Passwell JH et al. Safety and immunogenicity of Shigella sonneiCRM9 and Shigella flexneri type 2a-rEPAsucc conjugate vaccines in one-to four-year-old children. Pediatr Infect Dis J. 2003;22:701-6.

52. Riddle MS, Kaminski RW, Williams C, Porter C, Baqar S, Kordis A, Gilliland T, Lapa J, Coughlin M, Soltis C, Jones E, Saunders J, Keiser PB, Ranallo RT, Gormley R, Nelson M, Turbyfill KR, Tribble D, Oaks EV. Safety and immunogenicity of an intranasal Shigella flexneri 2a Invaplex 50 vaccine. Vaccine. 2011;29(40): 7009-19. A recent clinical trial that demonstrated the safety and immunogenicity of an LPS and Ipa-based Shigella vaccine delivered by intranasal spray.

53. Martinez-Becerra FJ, Kissmann JM, Diaz-McNair J, Choudhari SP, Quick AM, Mellado-Sanchez G, et al. Broadly Protective Shigella Vaccine Based on Type III Secretion Apparatus Proteins. Infect Immun. 2012;80:1222-31.

54. Khan WA, Griffiths JK, Bennish ML. Gastrointestinal and extraintestinal manifestations of childhood shigellosis in a region where all four species of Shigella are endemic. PLoS One. 2013;8:e64097. 
An in-depth comparison of the clinical manifestations and outcomes in children infected with different species of Shigella, demonstrating the greater virulence of $S$. dysenteriae strains.

55. Butler T. Haemolytic uraemic syndrome during shigellosis. Trans R Soc Trop Med. 2012;106:395-9.

56. Fischer-Walker CL, Applegate JA, Black RE. Haemolytic-uraemic syndrome as a sequel of diarrhoeal disease. J Health Popul Nutr. 2012;30:257-61.

57. Koster F, Levin J, Walker L, Tung KS, Gilman RH, Rahaman MM, et al. Hemolytic-uremic syndrome after shigellosis. Relation to endotoxemia and circulating immune complexes. N Engl J Med. 1978;298:927-33.

58. Bhimma R, Rollins NC, Coovadia HM, Adhikari M. Postdysenteric hemolytic uremic syndrome in children during an epidemic of Shigella dysentery in Kwazulu/Natal. Pediatr Nephrol. 1997;11:560-4.

59. Gu B, Cao Y, Pan S, Zhuang L, Yu R, Peng Z, et al. Comparison of the prevalence and changing resistance to nalidixic acid and ciprofloxacin of Shigella. Int J Antimicrob Agents. 2012;40:9-17. An extensive review of Shigella resistance trends worldwide that documents a 10-fold to 16-fold rate of quinolone resistance in the AsiaAfrica region compared to Europe and America.

60. Xia S, Xu B, Huang L, Zhao JY, Ran L, Zhang J, et al. Prevalence and characterization of human Shigella infections in Henan Province, China, in 2006. J Clin Microbiol. 2011;49:232-42.
61. Taneja N, Mewara A, Kumar A, Verma G, Sharma M. Cephalosporin-resistant Shigella flexneri over 9 years (2001-09) in India. J Antimicrob Chemother. 2012;67:1347-53.

62. Sabra AH, Araj GF, Kattar MM, Abi-Rached RY, Khairallah MT, Klena JD, et al. Molecular characterization of ESBLproducing Shigella sonnei isolates from patients with bacilliary dysentery in Lebanon. J Infect Dev Ctries. 2009;3: $300-5$.

63. Tajbakhsh M, García Migura L, Rahbar M, Svendsen CA, Mohammadzadeh M, Zali MR, et al. Antimicrobial-resistant Shigella infections from Iran: an overlooked problem? J Antimicrob Chemother. 2012;67:1128-33.

64. Salam MA, Seas C, Khan WA, Bennish ML. Treatment of shigellosis: IV: Cefixime is ineffective in adults. Ann Intern Med. 1995;123:505-8.

65. Martin JM, Pitetti R, Maffei F, Tritt J, Smail K, Wald ER. Treatment of shigellosis with cefixime: two days vs. five days. Pediatr Infect Dis J. 2000;19:522-6.

66. Greco KM, McDonough MA, Butterton JR. Variation in the Shiga toxin region of 20th-century epidemic and endemic Shigella dysenteriae 1 strains. J Infect Dis. 2004;190:330-4.

67. Bennish ML, Khan WA, Begum M, Bridges EA, Ahmed S, Saha D, et al. Low risk of hemolytic uremic syndrome after early effective antimicrobial therapy for Shigella dysenteriae Type 1 infection in Bangladesh. Clin Infect Dis. 2006;42:356-62. 